\title{
molecules
}

ISSN 1420-3049

(C) 2007 by MDPI

www.mdpi.org/molecules

Full Paper

\section{Synthesis of Sulfonamides and Evaluation of Their Histone Deacetylase (HDAC) Activity}

\author{
Seikwan Oh ${ }^{1}$, Hyung-In Moon ${ }^{2}$, Il-Hong Son ${ }^{2}$ and Jae-Chul Jung ${ }^{1, *}$ \\ ${ }^{1}$ Department of Neuroscience and Medical Research Institute, College of Medicine, Ewha Womans \\ University, Seoul 158-710, South Korea. \\ 2 Department of Neuroscience and Inam Neuro Science Research Center, Wonkwang University \\ Sanbon Medical Center, Sanbondong, Gunpocity, Kyunggido, 435-040, South Korea. E-mails: \\ himoon@wonkwang.ac.kr (Hyung-In Moon); soniag@wonkwang.ac.kr (Il-Hong Son)
}

* To whom correspondence should be addressed; jcjung@ewha.ac.kr

Received: 17 April 2007; in revised form: 18 May 2007 / Accepted: 18 May 2007 / Published: 24 May 2007

\begin{abstract}
A simple synthesis of sulfonamides 4-22 as novel histone deacetylase (HDAC) inhibitors is described. The key synthetic strategies involve $N$-sulfonylation of $L$-proline benzyl ester hydrochloride (2) and coupling reaction of $N$-sulfonyl chloride 3 with amines in high yields. It was found that several compounds showed good cellular potency with the most potent compound 20 exhibiting an $\mathrm{IC}_{50}=2.8 \mu \mathrm{M}$ in vitro.
\end{abstract}

Keywords: HDAC; $N$-Sulfonylation; Coupling reaction; Anticancer.

\section{Introduction}

Histone acetylation and deacetylation play fundamental roles in the modulation of chromatin topology and the regulation of gene transcription [1]. Histone deacetylase (HDAC) inhibitors that inhibit proliferation and induce differentiation and/or apoptosis of tumor cells in culture and in animal models have been identified [2]. A number of structurally diverse histone deacetylase inhibitors have shown potent antitumor efficacy with little toxicity in vivo in animal models. Recently, HDAC inhibitors have emerged as an exciting new class of potential anticancer agents for the treatment of solid and hematological malignancies [3]. Current research priorities are to better characterize the 
biological roles and biochemical features of HDAC inhibitors. In addition, efforts to identify optimal HDAC inhibitors for anticancer therapeutics are underway [4]. More recently, the Angibaud group [5] reported the preparation of a series of pyrimidyl-5-hydroxamic acids having significant HDAC activity in human tumor cell lines. The Kalvinsh group [6] demonstrated that a series of novel sulfonamide derivatives were synthesized and evaluated for their ability to inhibit human HDAC. The Delorme group [7] developed of potential antitumor agents as a new set of sulfonamide derivatives. The Trivedi group [8] described the QSAR modeling of sulfonamide inhibitors of HDAC.

In the context of our medicinal chemistry program dealing with the development of new potent anticancer agents, we required sulfonyl chloride $\mathbf{3}$ as a key fragment in order to generate novel HDAC inhibitors. We wish to report herein an efficient synthesis in good yields of sulfonamides 4-22, starting from $L$-proline (1) via benzylation, sulfonylation, and coupling reaction and the evaluation of their anti-proliferative inhibiting potency.

\section{Results and Discussion}

\section{Chemistry}

A series of sulfonamides 4-22 was prepared from $(L)$-proline (1) as a starting material, which was condensed with benzyl alcohol in the presence of thionyl chloride in dichloromethane to give $L_{-}$ proline benzyl ester hydrochloride (2) in 68\% yield [9].

Scheme 1. Synthesis of sulfonamides 4-7.

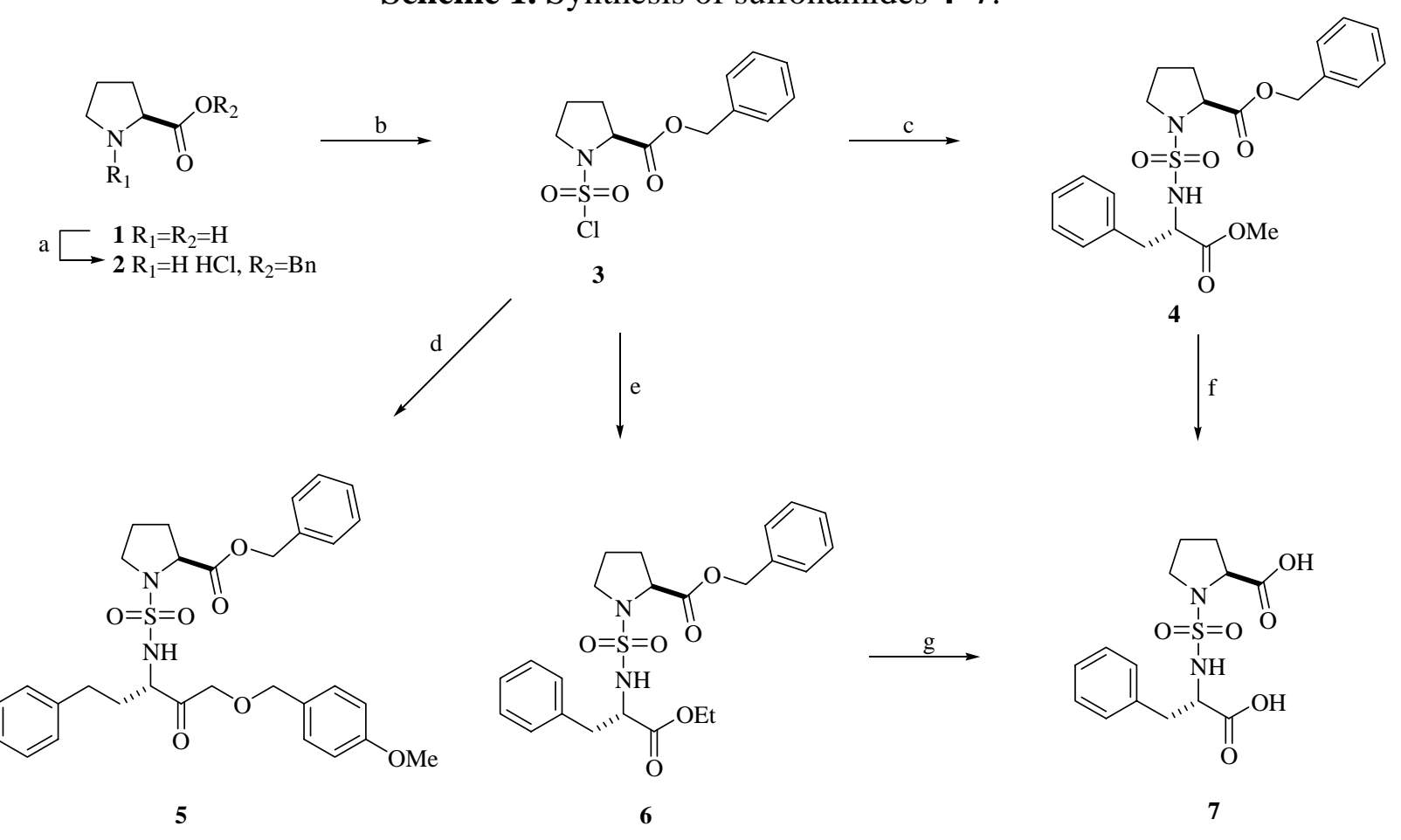

Benzyl alcohol, $\mathrm{SOCl}_{2}, \mathrm{CH}_{2} \mathrm{Cl}_{2}$, rt, $20 \mathrm{~h}$, (68\%); (b) $\mathrm{SO}_{2} \mathrm{Cl}_{2}$, TEA, 4-DMAP, toluene, $0{ }^{\circ} \mathrm{C}, 2 \mathrm{~h}$, (72\%); (c) L-phenylalanine methyl ester. $\mathrm{HCl}$, DIPEA, 4-DMAP, $\mathrm{CH}_{2} \mathrm{Cl}_{2}, \mathrm{rt}, 16 \mathrm{~h},(90 \%) ;$ (d) 10 , DIPEA, 4-DMAP, $\mathrm{CH}_{2} \mathrm{Cl}_{2}$, rt, 16 h, (80\%); (e) L-phenylalanine ethyl ester·HCl, DIPEA, 4DMAP, $\mathrm{CH}_{2} \mathrm{Cl}_{2}$, rt, 16 h, (93\%); (f) $\mathrm{LiOH}, \mathrm{THF} / \mathrm{H}_{2} \mathrm{O}, 0{ }^{\circ} \mathrm{C}$ to rt, $4 \mathrm{~h},(80 \%)$; (g) $1 N-\mathrm{NaOH} /$ EtOH, rt, 16 h, (71\%). 
Compound 2 was treated with sulfuryl chloride $\left(\mathrm{SO}_{2} \mathrm{Cl}_{2}\right)$ in toluene to generate in $72 \%$ yield the key intermediate 3 [10], which was subsequently coupled with $L$-phenylalanine methyl ester hydrochloride and $L$-phenylalanine ethyl ester hydrochloride in the presence of diisopropylethylamine (DIPEA) and 4-dimethylaminopyridine (4-DMAP) in dichloromethane to afford $\mathbf{4}$ and $\mathbf{6}$ in $90 \%$ and $93 \%$ yields, respectively.

Sulfonamides 4 and $\mathbf{6}$ were readily hydrolyzed by lithium hydroxide aqueous solution or $1 \mathrm{~N}$ sodium hydroxide aqueous solution to give acid 7 in $80 \%$ and $71 \%$ yields, respectively. In this hydrolysis reaction, basic hydrolysis $\left(1 N-\mathrm{NaOH} / \mathrm{MeOH}\right.$ or $\left.\mathrm{LiOH}, \mathrm{H}_{2} \mathrm{O}_{2}, \mathrm{THF} / \mathrm{H}_{2} \mathrm{O}\right)$ is more favorable than acidic hydrolysis $\left(3 N-\mathrm{HCl} / \mathrm{THF}-\mathrm{H}_{2} \mathrm{O}, \mathrm{TFA} / \mathrm{CH}_{2} \mathrm{Cl}_{2}\right)$ for preparation of acid $\mathbf{7}$ due to the higher yield and ease of handling. Furthermore, $\mathrm{LiOH}$ conditions afforded a superior yield for comparing with $1 N-\mathrm{NaOH}$ condition (Scheme 1).

Scheme 2. Synthesis of PMB amine 10.

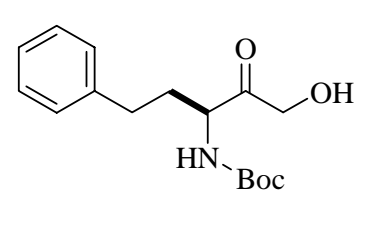

8

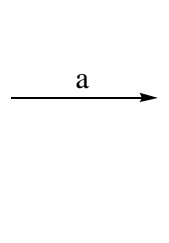

(1)

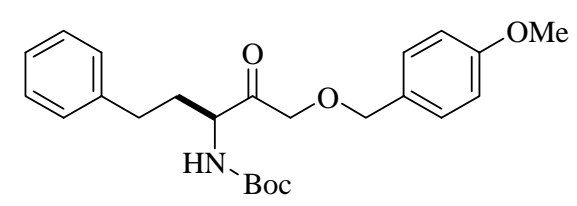

9
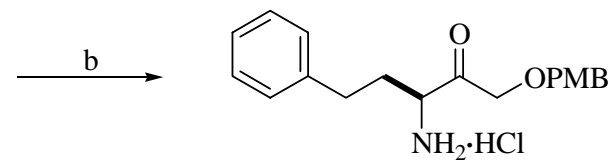

10

(a) 4-Methoxybenzyl-2,2,2-trichloroacetimidate, $\mathrm{Sc}(\mathrm{OTf})_{3}, \mathrm{CH}_{2} \mathrm{Cl}_{2}, 0{ }^{\circ} \mathrm{C}$ to rt, $20 \mathrm{~min}$, (97\%); (b) $3 N$-HCl, 1,4-dioxane $0{ }^{\circ} \mathrm{C}$ to rt, 2 h, (64\%).

To generate sulfonamide 5, PMB-amine 10 was prepared from compound 8 [11] which was protected with freshly prepared 4-methoxybenzyl-2,2,2-trichloroacetimidate (commercially available p-methoxybenzyl alcohol was treated with $\mathrm{Cl}_{3} \mathrm{CCN}$ in the presence of 1,5,7-triazabicyclo[4.4.0]dec-5ene) and catalytic amount of scandium triflate to give 9 [12], which was subsequently treated with $3 \mathrm{~N}$ $\mathrm{HCl}$ aqueous solution in 1,4-dioxane in order to removal of $N$-Boc group to thus afford $\mathbf{1 0}$ in $62 \%$ yield (over two steps) (Scheme 2). Interestingly, the condensation reaction of sulfonyl chloride 3 with $\mathbf{1 0}$ was took place smoothly to generate $\mathbf{5}$ in low yield. Unfortunately, compound $\mathbf{5}$ was unstable, and isolation and characterization were problematic.

Sulfonyl chloride 3 was then coupled with several amines [amines including aromatic rings; $(S)$ (+)-ethyl-4-phenylbutyrate-2-amine, 2-thiopheneethanamine, 4-fluorophenethylamine, pyridoxamine, $(R)-(+)-\alpha$-methylbenzylamine, and $(S)-(+)-\alpha$-(methoxymethyl)phenethylamine; amines including aliphatic groups; $N, N$-dimethylethylenediamine; 1-(2-aminoethyl)pyrrolidine, $(S)-(+)$-2-(hydroxylmethyl)pyrrolidine, $N$-(2-hydroxyethyl)piperazine, $N$-(2-aminoethyl)morpholine, and 2-(4-methylpiperazin-1-yl)-ethylamine] in the presence DIPEA and 4-DMAP in dichloromethane to give sulfonamides 11-22 in high yields (Scheme 3). 
Scheme 3. Synthesis of sulfonamides 11-22.<smiles>O=C(OCc1ccccc1)C1CCCN1S(=O)(=O)Cl</smiles>

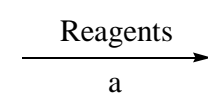

3

$11 \mathrm{R}=$<smiles>CCC(=O)C(=O)NC(C)Cc1ccccc1</smiles>

$12 \mathrm{R}=$<smiles>c1csc(CCC2CCCC2)c1</smiles><smiles>CC(C)NCCc1ccc(F)cc1</smiles><smiles>CCNc1c(CO)cnc(C)c1O</smiles><smiles>CC(C)c1ccccc1</smiles><smiles>COCC(c1ccccc1)N(C)C</smiles><smiles>CCNCCN(C)C</smiles>

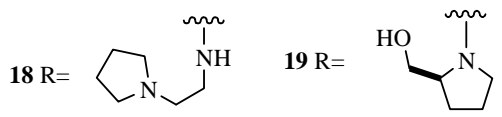

$20 \mathrm{R}=$<smiles>CN1CCN(CCO)CC1</smiles>

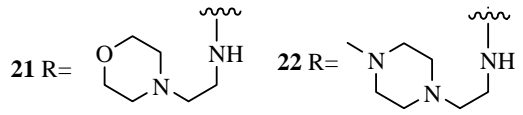

(a) Reagents, DIPEA, 4-DMAP, $\mathrm{CH}_{2} \mathrm{Cl}_{2}$, rt, 3-16 h, (80\%-95\%).

\section{Biological Activity}

The in vitro growth inhibiting potency of sulfonamides $\mathbf{4}$ and 6-22 were evaluated and the results are summarized in Table 1 . We found that potent inhibition was observed with piperazine-sulfonamide 20, while compounds 4, 6, 11-12, 15, 17-19, and 21 did not possess HDAC activities. When aromatic groups and pyrrolidine groups were used as coupling agents (compounds 4, 6, 11-12, 15, and 18-19), the resulting compounds exhibited significantly reduced HDAC activity. Interestingly, piperazinebased sulfonamides $\mathbf{2 0}$ and $\mathbf{2 1}$ were showed higher in vitro growth inhibiting potency when compared to pyrrolidine-based sulfonamides $\mathbf{1 8}$ and $\mathbf{1 9}$.

Table 1. HDAC and growth inhibiting potency of novel sulfonamides $\mathbf{4}$ and $\mathbf{6 - 2 2 .}$

\begin{tabular}{|c|c|}
\hline Compound & $\mathrm{IC}_{50}$ cells $(\mu \mathrm{M})^{\mathrm{a}}$ \\
\hline 7 & 40.3 \\
\hline 13 & 36.8 \\
\hline 14 & 25.3 \\
\hline 16 & 21.5 \\
\hline 20 & 2.8 \\
\hline 22 & 12.3 \\
\hline $4,6,11-12,15,17-19$, and 21 & $>100$ \\
\hline Sodium butyrate $^{\mathrm{b}}$ & 140 \\
\hline Trichostatin $\mathrm{A}^{\mathrm{c}}$ & 0.0046 \\
\hline
\end{tabular}

${ }^{\mathrm{a}}$ The values are means of three experiments.

${ }^{\mathrm{b}, \mathrm{c}}$ Reference materials. 


\section{Conclusions}

In conclusion, a simple preparation of novel histone deacetylase (HDAC) inhibitors has been described. The key synthetic strategies involve $O$-benzylation, $N$-sulfonylation, and coupling reactions carried out in high yields. Compound $\mathbf{2 0}$ exhibited the most potent HDAC activity among these analogues. We have found that piperazine-based sulfonamides $\mathbf{2 0}$ and $\mathbf{2 1}$ showed improved growth inhibiting potency in vitro. In addition, the novel sulfonamides 7, 13, 14, 16, 20 and 22) showed better HDAC activity than sodium butyrate. Although all prepared sulfonamides were exhibited less HDAC activity than trichostatin A as a compared material, we have expected that simple syntheses of new sulfonamide moieties and key fragments are useful for the modification of histone deacetylase (HDAC) inhibitors.

\section{Experimental}

\section{General}

Reactions requiring anhydrous conditions were performed with the usual precautions for rigorous exclusion of air and moisture. Tetrahydrofuran was distilled from sodium benzophenone ketyl prior to use. Thin layer chromatography (TLC) was performed on precoated silica gel G and GP uniplates from Analtech and visualized with 254-nm UV light. Flash chromatography was carried out on silica gel 60 [Scientific Adsorbents Incorporated (SAI), particle size $32-63 \mu \mathrm{m}$, pore size $60 \AA$ ]. ${ }^{1} \mathrm{H}-\mathrm{NMR}$ and ${ }^{13} \mathrm{C}$ NMR spectra were recorded on Bruker DPX 500 instrument at $500 \mathrm{MHz}\left({ }^{1} \mathrm{H}\right)$ and $125 \mathrm{MHz}\left({ }^{13} \mathrm{C}\right)$, respectively. The chemical shifts are reported in parts per million (ppm) downfield from tetramethylsilane. Infrared (IR) spectra were obtained on an ATI Mattson FT/IR spectrometer. Mass spectra were recorded with a Waters Micromass ZQ LC-Mass system and high resolution mass spectra (HRMS) were measured with a Bruker BioApex FTMS system by direct injection using an electrospray interface (ESI). Elemental analyses were performed on a CE instruments Model 1110 elemental analyzer. When necessary, chemicals were purified according to the reported procedures [13].

\section{Biology: In Vitro Inhibition of Histone Deacetylase}

Histone deacetylase fraction was prepared as described by Yoshida et al. [14]. Human leukemia K562 $\left(2.5 \times 10^{8}\right)$ cells were disrupted in buffer-A (15 mM potassium phosphate buffer, $\mathrm{pH} 7.5$, containing $5 \%$ glycerol and $0.2 \mathrm{mM}$ EDTA, $15 \mathrm{~mL}$ ). The nuclei were collected by centrifugation (35000g, $10 \mathrm{~min})$ and resuspended with buffer-A $(15 \mathrm{~mL})$ containing $1 \mathrm{M}\left(\mathrm{NH}_{4}\right)_{2} \mathrm{SO}_{4}$. After sonication, the supernatant was collected by centrifugation, and ammonium sulfate was added to make the final concentration $3.5 \mathrm{M}$. After stirring for $1 \mathrm{~h}$ at $0{ }^{\circ} \mathrm{C}$, the precipitate was collected by centrifugation, dissolved with buffer-A (4 mL), and dialyzed against buffer-A (2000 mL). The dialysate was loaded onto a mono Q HR 5/5 column (Pharmacia) equilibrated with buffer-A and eluted with a linear gradient of $0-1 \mathrm{M}-\mathrm{NaCl}$ in buffer-A $(30 \mathrm{~mL})$. A single peak of histone deacetylase activity was eluted around $0.4 \mathrm{M}-\mathrm{NaCl}$, and the fraction was stored at $-80{ }^{\circ} \mathrm{C}$ until use. Inhibition of 
histone deacetylase was estimated as described by Yoshida et al. with slight modifications [14]. 3HLabeled histone was prepared by the method of Yoshida et al.: $3 \mathrm{~K} 562$ cells (108 cells) were incubated in growth medium (25 mL) containing $0.5 \mathrm{mCi} / \mathrm{mL}$ [3H]sodium acetate $(152.8 \mathrm{GBq} / \mathrm{mmol}$; NEN) and $5 \mathrm{mM}$ sodium butyrate at $37^{\circ} \mathrm{C}$ [14]. Histone deacetylase inhibitory activity of test compound was measured as follows: the mixture (total volume $50 \mu \mathrm{L}$ ) containing the above histone deacetylase fraction $(2 \mu \mathrm{L})$, $3 \mathrm{H}$ labeled histone $(100 \mu \mathrm{g} / \mathrm{mL})$, and test compound $(5 \mu \mathrm{L})$ was incubated for $10 \mathrm{~min}$ at $37{ }^{\circ} \mathrm{C}$. [3H]Acetic acid, which was liberated from $3 \mathrm{H}$-labeled histone, was extracted with ethyl acetate, and radioactivity was measured by a liquid scintillation counter.

\section{Chlorosulfonyl-L-proline benzyl ester (3)}

To a stirred solution of $L$-proline benzyl ester hydrochloride (2, $2.4 \mathrm{~g}, 10.0 \mathrm{mmol}$ ) in dry toluene (25 mL) were added dropwise TEA (2.3 g, $21.0 \mathrm{mmol})$ and DMAP (0.12 g), followed by addition of sulfuryl dichloride $(2.7 \mathrm{~g}, 20.0 \mathrm{mmol})$ in dry toluene $(15 \mathrm{~mL})$ at $-10{ }^{\circ} \mathrm{C}$ and the mixture was stirred at $0{ }^{\circ} \mathrm{C}$ for $2 \mathrm{~h}$. The reaction mixture was diluted with dichloromethane $(70 \mathrm{~mL})$ and washed with sat'd. aqueous $\mathrm{NH}_{4} \mathrm{Cl}$ solution $(80 \mathrm{~mL})$ and water $(100 \mathrm{~mL})$. The organic layer was separated, dried over anhydrous $\mathrm{MgSO}_{4}$, filtered, and concentrated under reduced pressure. The residue was purified by flash column chromatography (silica gel, 15\% ethyl acetate in hexanes) to give 3 (1.45 g, 48\%) as a beige solid. $\mathrm{R}_{f}=0.4$ (15\% ethyl acetate in hexanes); $[\alpha]^{24} \mathrm{D}-105.0$ (c 0.6, $\mathrm{CHCl}_{3}$ ); mp $65{ }^{\circ} \mathrm{C}$ (lit. [15] $\mathrm{mp}$

65-66 ${ }^{\circ} \mathrm{C}$ ); IR (neat, $\left.\mathrm{NaCl}\right) 3474,3412,1638,1618,1457,1124,837 \mathrm{~cm}^{-1} ;{ }^{1} \mathrm{H}-\mathrm{NMR}\left(\mathrm{CDCl}_{3}\right) \delta 7.46-$ 7.32 (m, 5H), 5.24 (s, 2H), 4.47 (dd, $J=4.0,4.0 \mathrm{~Hz}, 1 \mathrm{H}), 3.79-3.73$ (m, 1H), 3.63-3.57 (m, 1H), $2.34-$ $2.31(\mathrm{~m}, 1 \mathrm{H}), 2.21-2.15$ (m, 1H), 2.13-2.06 (m, 2H); ${ }^{13} \mathrm{C}-\mathrm{NMR}\left(\mathrm{CDCl}_{3}\right) \delta 169.4,135.0,128.6,128.5$, 128.1, 67.8, 62.7, 51.7, 31.0, 24.8; HRMS calcd. for $\mathrm{C}_{12} \mathrm{H}_{14} \mathrm{NO}_{4} \mathrm{SCINa} 326.0230[\mathrm{M}+\mathrm{Na}]^{+}$, found 326.0240 .

General procedure for coupling reaction of chlorosulfonyl-L-proline benzyl ester (3) and several amines

To a solution of chlorosulfonyl- $L$-proline benzyl ester (3, $0.3 \mathrm{~g}, 1.0 \mathrm{mmol})$ in dichloromethane (10 $\mathrm{mL}$ ) were added DIPEA (0.19 g, $1.5 \mathrm{mmol})$ and DMAP (0.12 g, $0.1 \mathrm{mmol})$, followed by addition of amines $(1.1 \mathrm{mmol})$ at $0{ }^{\circ} \mathrm{C}$ and the reaction mixture was stirred at room temperature for $16 \mathrm{~h}$. The reaction mixture was diluted with dichloromethane $(10 \mathrm{~mL})$ and washed with sat'd aqueous $\mathrm{NH}_{4} \mathrm{Cl}$ solution $(10 \mathrm{~mL})$ and brine $(10 \mathrm{~mL})$. The organic layer was separated, dried over anhydrous $\mathrm{MgSO}_{4}$, filtered, and concentrated under reduced pressure. The residue was purified by flash column chromatography (silica gel, ethyl acetate-hexane-methanol; 15:80: 5, $v / v$ ) to afford sulfonyl-L-proline benzyl esters 4-22.

$N$-(L-Phenylalanine methyl ester)sulfonyl-L-proline benzyl ester (4). Yield: $90 \%$; viscous oil; $\mathrm{R}_{f}=0.3$ (80:15:5 $n$-hexane-ethyl acetate-methanol, $v / v$ ); $[\alpha]^{25}$ D -12.5 (c 0.40, $\mathrm{CHCl}_{3}$ ); IR (neat, $\mathrm{NaCl}$ ) 3474, 3412, 1638, 1618, 1457, 1124, $837 \mathrm{~cm}^{-1} ;{ }^{1} \mathrm{H}-\mathrm{NMR}\left(\mathrm{CDCl}_{3}\right) \delta 7.42-7.18(\mathrm{~m}, 10 \mathrm{H}), 5.20(\mathrm{~s}, 2 \mathrm{H}), 4.56$ (brs, 1H), 4.38 (dd, $J=4.0,4.0 \mathrm{~Hz}, 1 \mathrm{H}$ ), 3.75 (s, 3H), 3.74 (s, $1 \mathrm{H}$ ), 3.25 (dd, $J=7.0,7.0 \mathrm{~Hz}, 1 \mathrm{H}$ ), 3.13 
(dd, $J=5.5,5.5 \mathrm{~Hz}, 1 \mathrm{H}$ ), 3.00-2.87 (m, 2H), 2.20-2.14 (m, 1H), 2.00-1.93 (m, 1H), 1.85-1.80 (m, 2H); ${ }^{13} \mathrm{C}-\mathrm{NMR}\left(\mathrm{CDCl}_{3}\right) \delta 172.2,172.0,137.1,135.7,135.3,129.5,129.2,128.5,128.4,128.3,128.1$, 126.7, 67.3, 61.0, 57.0, 52.7, 48.3, 39.4, 31.2, 25.1; HRMS calcd. for $\mathrm{C}_{22} \mathrm{H}_{27} \mathrm{~N}_{2} \mathrm{O}_{6} \mathrm{~S}: 447.1590[\mathrm{M}+\mathrm{H}]^{+}$, found: 447.1609; Anal. calcd. C 59.27, H 5.76, N 6.38; found: C 59.18, H 5.87, N 6.27.

$N$-(L-Phenylalanine ethyl ester)sulfonyl-L-proline benzyl ester (6). Yield: 93\%; viscous oil; $\mathrm{R}_{f}=0.4$ (80:15:5 n-hexane-ethyl acetate-methanol, $v / v$ ); $[\alpha]^{25}{ }_{\mathrm{D}}-105.2$ (c 1.5, $\mathrm{CHCl}_{3}$ ); IR (neat, $\mathrm{NaCl}$ ) 3288, 3064, 3029, 2981, 1740, 1604, 1455, 1340, 1151, 1020, $859 \mathrm{~cm}^{-1} ;{ }^{1} \mathrm{H}-\mathrm{NMR}\left(\mathrm{CDCl}_{3}\right) \delta$ 7.41-7.18 (m, $10 \mathrm{H}$ ), 5.41 (brs, $1 \mathrm{H}), 5.21$ (dd, $J=8.0,8.0 \mathrm{~Hz}, 2 \mathrm{H}), 4.47$ (dd, $J=4.0,4.0 \mathrm{~Hz}, 1 \mathrm{H}), 4.30-4.23$ (m, $1 \mathrm{H}$ ), 4.21 (q, $J=4.5 \mathrm{~Hz}, 2 \mathrm{H}), 3.52-3.41(\mathrm{~m}, 2 \mathrm{H}), 2.84-2.65(\mathrm{~m}, 2 \mathrm{H}), 2.32-2.23(\mathrm{~m}, 1 \mathrm{H}), 2.22-2.12$ (m, 1H), $2.08-1.90(\mathrm{~m}, 4 \mathrm{H}), 1.32(\mathrm{t}, J=7.0 \mathrm{~Hz}, 3 \mathrm{H}) ;{ }^{13} \mathrm{C}-\mathrm{NMR}\left(\mathrm{CDCl}_{3}\right) \delta 172.3,172.1,140.6,135.3$, 128.5, 128.4, 128.3, 128.1, 126.1, 67.3, 61.9, 61.4, 56.0, 48.6, 35.2, 31.7, 31.2, 25.2, 14.6; HRMS calcd. for $\mathrm{C}_{24} \mathrm{H}_{30} \mathrm{~N}_{2} \mathrm{O}_{6} \mathrm{SNa}$ : $497.1722[\mathrm{M}+\mathrm{Na}]^{+}$, found: 497.1743.

$N$-[(S)-Ethly 4-phenylbutanoate)sulfamoyl]-L-proline benzyl ester (11). Yield: 87\%; viscous oil; $\mathrm{R}_{f}=0.3$ (80:15:5 n-hexane-ethyl acetate-methanol, $\left.v / v\right) ;[\alpha]^{25}-105.2$ (c 0.5, $\mathrm{CHCl}_{3}$ ); IR (neat, $\mathrm{NaCl}$ ) 3288, 3064, 3029, 2981, 1740, 1604, 1455, 1340, 1151, 1020, $859 \mathrm{~cm}^{-1}{ }^{1}{ }^{1} \mathrm{H}-\mathrm{NMR}\left(\mathrm{CDCl}_{3}\right) \delta 7.41-7.18$ (m, 10H), 5.41 (brs, $1 \mathrm{H}$ ), 5.21 (dd, $J=8.0,8.0 \mathrm{~Hz}, 2 \mathrm{H}$ ), 4.47 (dd, $J=4.0,4.0 \mathrm{~Hz}, 1 \mathrm{H}$ ), 4.30-4.23 (m, $1 \mathrm{H}), 4.21$ (q, $J=4.5 \mathrm{~Hz}, 2 \mathrm{H}), 3.52-3.41(\mathrm{~m}, 2 \mathrm{H}), 2.84-2.65(\mathrm{~m}, 2 \mathrm{H}), 2.32-2.23(\mathrm{~m}, 1 \mathrm{H}), 2.22-2.12$ (m, 1H), 2.08-1.90 (m, 4H), $1.32(\mathrm{t}, J=7.0 \mathrm{~Hz}, 3 \mathrm{H}) ;{ }^{13} \mathrm{C}-\mathrm{NMR}\left(\mathrm{CDCl}_{3}\right) \delta 172.3,172.1,140.6,135.3$, 128.5, 128.4, 128.3, 128.1, 126.1, 67.3, 61.9, 61.4, 56.0, 48.6, 35.2, 31.7, 31.2, 25.2, 14.6; HRMS calcd. for $\mathrm{C}_{24} \mathrm{H}_{30} \mathrm{~N}_{2} \mathrm{O}_{6} \mathrm{SNa}$ : 497.1722 [M+Na] $]^{+}$, found: 497.1743 .

1-[2-Thiophenethylsulfamoyl)]-L-proline benzyl ester (12). Yield: 90\%; viscous oil; $\mathrm{R}_{f}=0.3$ (80:15:5 $n$-hexane-ethyl acetate-methanol, $v / v)$; $[\alpha]^{25}{ }_{\mathrm{D}}-34.8$ (c 0.5, $\mathrm{CHCl}_{3}$ ); IR (neat, $\left.\mathrm{NaCl}\right) 3302,3067,3034$, 2954, 2881, 1743, 1638, 1455, 1330, 1148, 1017, $849 \mathrm{~cm}^{-1} ;{ }^{1} \mathrm{H}-\mathrm{NMR}\left(\mathrm{CDCl}_{3}\right) \delta 7.52-7.30(\mathrm{~m}, 5 \mathrm{H})$, 7.24-7.14 (m, 1H), 7.00-6.83 (m, 2H), 5.29-5.11 (m, 2H), 4.75-4.60 (m, 1H), 4.52-4.42 (m, 1H), 3.53-3.28 (m, 4H), 3.04 (t, $J=6.5 \mathrm{~Hz}, 2 \mathrm{H}), 2.36-2.24(\mathrm{~m}, 1 \mathrm{H}), 2.12-1.90$ (m, 3H); ${ }^{13} \mathrm{C}-\mathrm{NMR}\left(\mathrm{CDCl}_{3}\right)$ $\delta$ 172.5, 140.5, 135.3, 128.6, 128.4, 128.3, 127.0, 125.6, 124.0, 67.3, 61.3, 48.7, 44.7, 31.3, 30.6, 25.2; HRMS calcd. for $\mathrm{C}_{18} \mathrm{H}_{22} \mathrm{~N}_{2} \mathrm{O}_{4} \mathrm{~S}_{2} \mathrm{Na}$ : 417.0919 [M+Na] $]^{+}$, found: 417.0927; Anal. calcd. C 54.80, $\mathrm{H}$ 5.62, N 7.10; found: C 54.66, H 5.88, N 6.96.

1-[2-(4-Fluorophenethylsulfamoyl)]-L-proline benzyl ester (13). Yield: 85\%; viscous oil; $\mathrm{R}_{f}=0.3$ (80:15:5 n-hexane-ethyl acetate-methanol, $v / v$ ); $[\alpha]^{25}-31.2$ (c 0.25, $\mathrm{CHCl}_{3}$ ); IR (neat, $\mathrm{NaCl}$ ) 3297, 3067, 3036, 2955, 2881, 1747, 1602, 1510, 1455, 1328, 1219, 1149, 1076, $834 \mathrm{~cm}^{-1}$; ${ }^{1} \mathrm{H}-\mathrm{NMR}$ $\left(\mathrm{CDCl}_{3}\right) \delta 7.41-7.32(\mathrm{~m}, 5 \mathrm{H}), 7.20-7.12(\mathrm{~m}, 2 \mathrm{H}), 7.03-6.95(\mathrm{~m}, 2 \mathrm{H}), 5.29(\mathrm{dd}, J=12.0,12.0 \mathrm{~Hz}$, $2 \mathrm{H}), 4.62(\mathrm{t}, J=6.0 \mathrm{~Hz}, 1 \mathrm{H}), 4.45$ (dd, $J=4.0,4.0 \mathrm{~Hz}, 1 \mathrm{H}), 3.46-3.38(\mathrm{~m}, 1 \mathrm{H}), 3.37-3.25(\mathrm{~m}, 3 \mathrm{H})$, $2.80(\mathrm{t}, J=7.0 \mathrm{~Hz}, 2 \mathrm{H}), 2.32-2.21(\mathrm{~m}, 1 \mathrm{H}), 2.07-2.00(\mathrm{~m}, 1 \mathrm{H}), 1.98-1.90(\mathrm{~m}, 2 \mathrm{H}) ;{ }^{13} \mathrm{C}-\mathrm{NMR}\left(\mathrm{CDCl}_{3}\right)$ $\delta 172.5,162.4,160.5,135.3,134.0,130.2,128.5,128.3,128.1,115.4,115.3,67.2,61.2,48.7$, 44.6, 35.5, 31.3, 25.2; HRMS calcd. for $\mathrm{C}_{20} \mathrm{H}_{23} \mathrm{~N}_{2} \mathrm{O}_{4} \mathrm{SFNa}$ : 429.1260 [M+Na] $]^{+}$found: 429.1272; Anal. calcd. C 59.10, H 5.70, N 6.89; found: C 59.21, H 5.83, N 6.72. 
1-[(R)-Methylbenzylsulfamoyl]-L-proline benzyl ester (15). Yield: 93\%; viscous oil; $\mathrm{R}_{f}=0.3$ (80:15:5 $n$-hexane-ethyl acetate-methanol, $v / v)$; $[\alpha]^{25}{ }_{\mathrm{D}}-17.5$ (c 0.4, $\mathrm{CHCl}_{3}$ ); IR (neat, $\left.\mathrm{NaCl}\right) 3288,3032,2977$, 2879, 1747, 1605, 1497, 1332, 1151, 1084, $873 \mathrm{~cm}^{-1} ;{ }^{1} \mathrm{H}-\mathrm{NMR}\left(\mathrm{CDCl}_{3}\right) \delta 7.40-7.25$ (m, 10H), 5.20 (dd, $J=12.0,12.0 \mathrm{~Hz}, 2 \mathrm{H}), 5.14-5.05(\mathrm{~m}, 1 \mathrm{H}), 4.73-4.65(\mathrm{~m}, 1 \mathrm{H}), 4.40$ (dd, $J=4.0,4.0 \mathrm{~Hz}, 1 \mathrm{H}$ ), 3.31-3.19 (m, 2H), 2.16-2.07 (m, 1H), 2.01-1.94 (m, 1H), 1.92-1.76 (m, 2H), 1.53 (s, 3/2H), 1.51 (s, 3/2H); ${ }^{13} \mathrm{C}-\mathrm{NMR}\left(\mathrm{CDCl}_{3}\right) \delta 172.5,143.4,135.4,128.5,128.3,128.1,127.4,126.1,67.2,60.9,53.6$, 48.7, 31.3, 25.0, 24.2; HRMS calcd. for $\mathrm{C}_{20} \mathrm{H}_{24} \mathrm{~N}_{2} \mathrm{O}_{4} \mathrm{SNa}$ : 411.1354 [M+Na] ${ }^{+}$, found: 411.1367 .

1-[(1S)-Methoxymethyl-2-phenethylsulfamoyl]-L-proline benzyl ester (16). Yield: 89\%; viscous oil; $\mathrm{R}_{f}$ $=0.3$ (80:15:5 n-hexane-ethyl acetate-methanol, $v / v$ ); $[\alpha]_{\mathrm{D}}^{25}-67.3$ (c 0.30, $\mathrm{CHCl}_{3}$ ); IR (neat, $\mathrm{NaCl}$ ) 3288, 3030, 2980, 2892, 1749, 1603, 1498, 1331, 1149, 1083, $854 \mathrm{~cm}^{-1}{ }^{1}{ }^{1} \mathrm{H}-\mathrm{NMR}\left(\mathrm{CDCl}_{3}\right) \delta 7.41-7.21$ (m, 10H), 5.20 (dd, $J=12.0,12.0 \mathrm{~Hz}, 2 \mathrm{H}), 4.76$ (d, $J=9.0 \mathrm{~Hz}, 1 \mathrm{H}), 4.40$ (dd, $J=4.5,4.5 \mathrm{~Hz}, 1 \mathrm{H})$, 3.90-3.81 (m, 1H), ), 3.44 (dd, $J=4.5,4.5 \mathrm{~Hz}, 1 \mathrm{H}$ ), 3.37 (s, 3H), 3.31 (dd, $J=3.0,3.0 \mathrm{~Hz}, 1 \mathrm{H}$ ), 3.22 (dd, $J=8.5,8.5 \mathrm{~Hz}, 1 \mathrm{H}), 2.96-2.84(\mathrm{~m}, 3 \mathrm{H}), 2.24-2.16(\mathrm{~m}, 1 \mathrm{H}), 2.01-1.94(\mathrm{~m}, 1 \mathrm{H}), 1.86-1.79$ (m, 2H); ${ }^{13} \mathrm{C}-\mathrm{NMR}\left(\mathrm{CDCl}_{3}\right) \delta 172.4,137.9,135.5,129.6,128.5,128.3,128.2,128.1,126.4,73.2,67.1$, 61.0, 59.2, 54.9, 48.3, 38.7, 31.3, 25.3; HRMS calcd. for $\mathrm{C}_{22} \mathrm{H}_{28} \mathrm{~N}_{2} \mathrm{O}_{5} \mathrm{SNa}$ : 455.1617 [M+Na] ${ }^{+}$, found: 455.1623; Anal. calcd. C 61.09, H 6.52, N 6.48; found: C 60.95, H 6.41, N 6.37.

1-[4-(S)-2-Hydroxymethyl)pyrrolidine-1-sulfonyl]-L-proline benzyl ester (19). Yield: 80\%; viscous oil; $\mathrm{R}_{f}=0.3$ (80:15:5 n-hexane-ethyl acetate-methanol, $\left.v / v\right)$; $[\alpha]^{25}-62.3$ (c 0.6, $\mathrm{CHCl}_{3}$ ); IR (neat, $\mathrm{NaCl}$ ) 3519, 3064, 3034, 2957, 2881, 1746, 1655, 1456, 1337, 1147, 1017, $826 \mathrm{~cm}^{-1}$; ${ }^{1} \mathrm{H}-\mathrm{NMR}\left(\mathrm{CDCl}_{3}\right)$

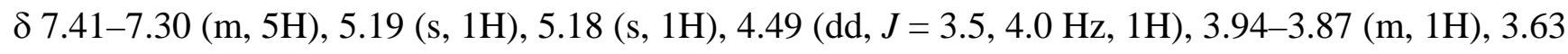
(dd, $J=4.5,4.5 \mathrm{~Hz}, 1 \mathrm{H}), 3.60-3.39$ (m, 4H), 3.31-3.22 (m, 1H), 2.73 (brs, 1H), 2.32-2.21 (m, 1H), 2.06-1.93 (m, 4H), 1.89-1.75 (m, 3H); ${ }^{13} \mathrm{C}-\mathrm{NMR}\left(\mathrm{CDCl}_{3}\right) \delta 172.4,135.3,128.6,128.5,128.4,128.3$, 128.2, 128.0, 67.3, 65.5, 61.8, 61.2, 50.1, 49.4, 31.4, 29.3, 25.3, 25.0; HRMS calcd. for $\mathrm{C}_{17} \mathrm{H}_{24} \mathrm{~N}_{2} \mathrm{O}_{5} \mathrm{SNa}$ : $391.1304[\mathrm{M}+\mathrm{Na}]^{+}$, found: 391.1299.

1-[4-(2-Hydroxyethyl)piperazine-1-sulfonyl]-L-proline benzyl ester (20). Yield: 81\%; viscous oil; $\mathrm{R}_{f}=0.4$ (80:15:5 n-hexane-ethyl acetate-methanol, $v / v$ ); $[\alpha]^{25}{ }_{\mathrm{D}}-35.7$ (c 0.60, $\mathrm{CHCl}_{3}$ ); IR (neat, $\mathrm{NaCl}$ ) 3416, 3065, 3034, 2950, 2879, 2821, 1747, 1455, 1340, 1154, 1016, $875 \mathrm{~cm}^{-1} ;{ }^{1} \mathrm{H}-\mathrm{NMR}\left(\mathrm{CDCl}_{3}\right)$ $\delta 7.40-7.30$ (m, 5H), 5.17 (d, $J=4.0 \mathrm{~Hz}, 2 \mathrm{H}), 4.42$ (dd, $J=3.5,3.5 \mathrm{~Hz}, 1 \mathrm{H}), 3.62(\mathrm{t}, J=5.5 \mathrm{~Hz}, 2 \mathrm{H})$, 3.57-3.44 (m, 1H), 3.43-3.36 (m, 1H), 3.32-3.19 (m, 4H), 2.53 (t, $J=5.5 \mathrm{~Hz}, 2 \mathrm{H}), 2.50-2.41$ (m, 5H), $2.30-2.20$ (m, 1H), 2.04-1.92 (m, 3H); ${ }^{13} \mathrm{C}-\mathrm{NMR}\left(\mathrm{CDCl}_{3}\right) \delta 172.4,135.4,128.5,128.3,128.0$, 67.1, 67.0, 61.1, 57.0, 53.4, 48.8, 39.4, 31.4, 25.3; HRMS calcd. for $\mathrm{C}_{18} \mathrm{H}_{28} \mathrm{~N}_{3} \mathrm{O}_{5} \mathrm{~S}: 398.1750[\mathrm{M}+\mathrm{H}]^{+}$, found: 398.1762; Anal. calcd. C 54.39, H 6.85, N 10.57; found: C 54.17, H 6.67, N 10.38

1-[2-(4-Methylpiperazin-1-yl)ethylsulfamoyl]-L-proline benzyl ester (22). Yield: 88\%; viscous oil; $\mathrm{R}_{f}=0.3$ (80:15:5 n-hexane-ethyl acetate-methanol, $v / v$ ); $[\alpha]_{\mathrm{D}}^{25}-34.0$ (c 0.60, $\mathrm{CHCl}_{3}$ ); IR (neat, $\mathrm{NaCl}$ ) 3286, 3064, 3034, 2956, 2817, 1745, 1455, 1330, 1148, 1012, $863 \mathrm{~cm}^{-1}$; ${ }^{1} \mathrm{H}-\mathrm{NMR}\left(\mathrm{CDCl}_{3}\right) \delta 7.39-7.31$ (m, 5H), 5.17 (dd, $J=12.5,12.5 \mathrm{~Hz}, 2 \mathrm{H}), 4.45$ (dd, $J=4.0,4.0 \mathrm{~Hz}, 1 \mathrm{H}), 3.52-3.37$ (m, 2H), 3.31-3.20 (m, 1H), 3.19-3.11 (m, 1H), 3.10-2.98 (m, 1H), 2.64-2.33 (m, 10H), 2.29 (s, 3H), 2.27-2.21 (m, 1H), 2.07-1.94 (m, 3H); ${ }^{13} \mathrm{C}-\mathrm{NMR}\left(\mathrm{CDCl}_{3}\right) \delta 172.4,135.4,128.5,128.4,128.3,128.0,127.3,126.8,67.1$, 
61.0, 56.4, 55.1, 52.6, 48.8, 39.7, 31.4, 25.3; HRMS calcd. for $\mathrm{C}_{19} \mathrm{H}_{31} \mathrm{~N}_{4} \mathrm{O}_{4} \mathrm{~S}$ : $411.2066[\mathrm{M}+\mathrm{H}]^{+}$, found: 411.2073; Anal. calcd. C 55.59 H 7.37, N 13.65; found: C 55.71, H 7.58, N 13.31.

\section{Acknowledgements}

This work has been supported by the KOSEF Brain Neurobiology Grant (2006) and Ewha Global Challenge (BK21) grant.

\section{References}

1. (a) Vigushin, D. M.; Coombes, R. C. Histone deacetylase inhibitors in cancer treatment. AntiCancer Drugs 2002, 13, 1-13; (b) Vigushin, D. M.; Coombes, R. C. Targeted histone deacetylase inhibition for cancer therapy. Curr. Cancer Drug Targ. 2004, 4, 205-218.

2. (a) Mai, A.; Massa, S.; Rotili, D.; Cerbara, I.; Valente, S.; Pezzi, R.; Simeoni, S.; Ragno, R. Histone deacetylation in epigenetics: an attractive target for anticancer therapy. Med. Res. Rev. 2005, 25, 261-309; (b) Dangond, F.; Henriksson, M.; Zardo, G.; Caiafa, P.; Ekstrom, T. J.; Gray, S. G. Differential expression of class I HDACs: roles of cell density and cell cycle. Int. J. Oncol. 2001, 19, 773-777.

3. (a) Imre, G.; Gekeler, V.; Leja, A.; Beckers, T.; Boehm, M. Histone deacetylase inhibitors suppress the inducibility of nuclear factor-kappaB by tumor necrosis factor-alpha receptor-1 down-regulation. Cancer Res. 2006, 66, 5409-5418; (b) O'Connor, O. A.; Heaney, M. L.; Schwartz, L.; Richardson, S.; Willim, R.; MacGregor-Cortelli, B.; Curly, T.; Moskowitz, C.; Portlock, C.; Horwitz, S.; Zelenetz, A. D.; Frankel, S.; Richon, V.; Marks, P.; Kelly, W. K. Clinical experience with intravenous and oral formulations of the novel histone deacetylase inhibitor suberoylanilide hydroxamic acid in patients with advanced hematologic malignancies. $J$. Clin. Oncol. 2006, 24, 166-173; (c) Mayo, M. W.; Denlinger C. E.; Broad R. M.; Yeung, F.; Reilly, E. T.; Shi, Y.; Jones, D. R. Ineffectiveness of histone deacetylase inhibitors to induce apoptosis involves the transcriptional activation of NF-kappa B through the Akt pathway. J. Biol. Chem. 2003, 278, 18980-18989.

4. (a) Suzuki, T.; Nagano, Y.; Kouketsu, A.; Matsuura, A.; Maruyama, S.; Kurotaki, M.; Nakagawa, H.; Miyata, N. Novel inhibitors of human histone deacetylases: design, synthesis, enzyme inhibition, and cancer cell growth inhibition of SAHA-based non-hydroxamates. J. Med. Chem. 2005, 48, 1019-1032; (b) Bouchain, G.; Delorme, D. Novel hydroxamate and anilide derivatives as potent histone deacetylase inhibitors: synthesis and antiproliferative evaluation. Curr. Med. Chem. 2003, 10, 2359-2372; (c) Shinji, C.; Maeda, S.; Imai, K.; Yoshida, M.; Hashimoto, Y.; Miyachi, H. Design, synthesis, and evaluation of cyclic amide/imide-bearing hydroxamic acid derivatives as class-selective histone deacetylase (HDAC) inhibitors. Bioorg. Med. Chem. 2006, 14, 7625-7651; (d) Kim, H. M.; Lee, K.; Park, B. W.; Ryu, D. K.; Kim, K.; Lee, C. W.; Park, S. K.; Han, J. W.; Lee, H. Y.; Lee, H, Y.; Han, G. Synthesis, enzymatic inhibition, and cancer cell growth inhibition of novel $\delta$-lactam-based histone deacetylase (HDAC) inhibitors. Bioorg. Med. Chem. Lett. 2006, 16, 4068-4070; (e) Fournel, M.; Trachy-Bourget, M. C.; Yan, P. T.; Kalita, A.; Bonfils, C.; Beaulieu, C.; Frechette, S.; Leit, S.; Abou-Khalil, E.; Woo, S. H.; Delorme, D.; MacLeod, A. R.; Besterman, J. M.; Li, Z. Sulfonamide anilides, a novel class of histone 
deacetylase inhibitors, are antiproliferative against human tumors. Cancer Res. 2002, 62, 43254330; (f) Owa, T.; Yoshino, H.; Yoshimatsu, K.; Nagasu, T. Cell cycle regulation in the G1 phase: a promising target for the development of new chemotherapeutic anticancer agents. Curr. Med. Chem. 2001, 8, 1487-1503; (g) Bouchain, G.; Delorme, D. Novel hydroxamate and anilide derivatives as potent histone deacetylase inhibitors: synthesis and antiproliferative evaluation. Curr. Med. Chem. 2003, 10, 2359-2372; (h) Uesato, S.; Kitagawa, M.; Nagaoka, Y.; Maeda, T.; Kuwajima, H.; Yamori, T. Novel histone deacetylase inhibitors: N-hydroxycarboxamides possessing a terminal bicyclic aryl group. Bioorg. Med. Chem. Lett. 2002, 12, 1347-1349; (i) Lavoie, R.; Bouchain, G.; Frechette, S.; Woo, S. H.; Abou-Khalil, E.; Leit, S.; Fournel, M.; Yan, P. T.; Trachy-Bourget, M. C.; Beaulieu, C.; Li, Z.; Besterman, J.; Delorme, D. Design and synthesis of a novel class of histone deacetylase inhibitors. Bioorg. Med. Chem. Lett. 2001, 11, 2847-2850.

5. Angibaud, P.; Arts, J.; Van Emelen, K.; Poncelet, V.; Pilatte, I.; Roux, B.; Van Brandt, S.; Verdonck, M.; De Winter, H.; Ten Holte, P.; Marien, A.; Floren, W.; Janssens, B.; Van Dun, J.; Aerts, A.; Van Gompel, J.; Gaurrand, S.; Queguiner, L.; Argoullon, J. M.; Van Hijfte, L.; Freyne, E.; Janicot, M. Discovery of pyrimidyl-5-hydroxamic acids as new potent histone deacetylase inhibitors. Eur. J. Med. Chem. 2005, 40, 597-606.

6. Finn, P. W.; Bandara, M.; Butcher, C.; Finn, A.; Hollinshead, R.; Khan, N.; Law, N.; Murthy, S.; Romero, R.; Watkins, C.; Andrianov, V.; Bokaldere, R. M.; Dikovska, K.; Gailite, V.; Loza, E.; Piskunova, I.; Starchenkov, I.; Vorona, M.; Kalvinsh, I. Novel sulfonamide derivatives as inhibitors of histone deacetylase. Helv. Chim. Acta 2005, 88, 1630-1657.

7. Bouchain, G.; Leit, S.; Frechette, S.; Khalil, E. A.; Lavoie, R.; Moradei, O.; Woo, S. H.; Fournel, M.; Yan, P. T.; Kalita, A.; Trachy-Bourget, M. C.; Beaulieu, C.; Li, Z.; Robert, M. F.; MacLeod, A. R.; Besterman, J. M.; Delorme, D. Development of potential antitumor agents. Synthesis and biological evaluation of a new set of sulfonamide derivatives as histone deacetylase inhibitors. $J$. Med. Chem. 2003, 46, 820-830.

8. Jaiswal, D.; Karthikeyan, C.; Shrivastava, S. K.; Trivedi, P. QSAR Modeling of Sulfonamide Inhibitors of Histone Deacetylase. Internet Electron. J. Mol. Des. 2006, 5, 345-354.

9. (a) Cordova, A.; Reed, N. N.; Ashley, J. A.; Janda, K. D. Convenient synthesis of L-proline benzyl ester. Bioorg. Med. Chem. Lett. 1999, 9, 3119-3122; (b) Cheeseright, T. J.; Edwards, A. J.; Elmore, D. T.; Jones, J. H.; Raissi, M.; Lewis, E. C. Azasulfonamidopeptides as peptides bond hydrolysis transition state analogues. Part I: Synthetic approaches. J. Chem. Soc. Perkin Trans 1 1994, 12, 1595-1600.

10. Berredjem, M.; Winum, J. Y.; Toupet, L.; Masmoudi, O.; Aouf, N. E.; Montero, J. L. NChlorosulfonyloxazolidin-2-ones: Synthesis, Structure, and Reactivity Toward Aminoesters. Synth. Commun. 2004, 34, 1653-1662.

11. (a) Okada, Y.; Tsukatani, M.; Taguchi, H.; Yokoi, T.; Bryant, S. D.; Lazarus, L. H. Amino acids and peptides. LII. Design and synthesis of opioid mimetics containing a pyrazinone ring and examination of their opioid receptor binding activity. Chem. Pharm. Bull. 1998, 46, 1374-1382; (b) Jung, J. C.; Avery, M. A. An efficient synthesis of cyclic urethanes from Boc-protected amino acids through a metal triflate-catalyzed intramolecular diazo carbonyl insertion reaction. Tetrahedron Lett. 2006, 47, 7969-7972. 
12. Reddy, C. R.; Chittiboyina, A. G.; Kache, R.; Jung, J. C.; Watkins, E. B.; Avery, M. A. The trimethylsilyl xylyl (TIX) ether: a useful protecting group for alcohols. Tetrahedron 2005, 61, 1289-1295.

13. Perrin, D. D.; Armarego, L. F.; Perrin, D. R. Purification of Laboratory Chemicals, $2^{\text {nd }}$ ed; Pergamon Press: New York, 1980.

14. (a) Finney, D. J. Graded Responses. In Probit Analyses, $2^{\text {nd }}$ ed.; Cambridge University Press: Cambridge, 1962; Chapter 10; (b) Yoshida, M.; Kijima, M.; Akita, M.; Beppu, T. Potent and specific inhibition of mammalian histone deacetylase both in vivo and in vitro by trichostatin A. $J$. Biol. Chem. 1990, 265, 17174-17179.

15. Cheeseright, T. J.; Daenke, S.; Elmore, D. T.; Jones, J. H. Azasulfonamidopeptides as peptide bond hydrolysis transition state analogues. Part 2. Potential HIV-1 proteinase inhibitor. J. Chem. Soc. Perkin Trans 1, 1994, 14, 1953-1955.

Sample Availability: Samples of the compounds are available from authors.

(c) 2007 by MDPI (http://www.mdpi.org). Reproduction is permitted for noncommercial purposes. 